\title{
Galactic outflows at high spatial resolution via gravitational lensing
}

\author{
Justin Spilker(1) \\ University of Texas at Austin, 2515 Speedway Stop C1400, Austin, TX 78712, USA \\ email: spilkerj@gmail.com
}

\begin{abstract}
The completion of the Atacama Large Millimeter/submillimeter Array (ALMA) has led to the ability to make observations with unprecedented resolution at sub-millimeter wavelengths, allowing novel probes of the ISM and kinematics of high-redshift galaxies. Because they are magnified by foreground galaxies or clusters, gravitationally lensed galaxies allow the highest possible spatial resolution to be obtained, and/or a sharp reduction in the observing time required to detect faint objects or spectral lines. These benefits have made lensed galaxies useful benchmark systems for ALMA, enabling a wide variety of science cases. Here I focus in particular on spatially-resolved observations of massive galactic outflows in the very distant $z>4$ universe, summarizing plausible tracers of the cold molecular phase of these outflows. The prospects of joint JWST and ALMA observations will be revolutionary, including the chance to take a full census of galactic outflows in multiple gas phases at matched spatial resolution.
\end{abstract}

Keywords. galaxies: high-redshift, galaxies: formation, galaxies: ISM

\section{Galactic Feedback and Outflows}

Self-regulating galactic feedback is a critical component of galaxy evolution, yet one that has remained difficult to probe observationally. For simple models in which only gravitation plays an important role in the growth of galaxies, virtually all baryonic mass collapses into stars very early in the history of the universe, yielding a population of galaxies far more massive and older than those observed in the real universe. Some energetic processes must be invoked to limit the growth rate of galaxies, by heating, depleting, expelling, or increasing the turbulence in the gas from which stars form. Energy and momentum from active galactic nuclei (AGN), Type II supernovae, and/or massive star winds and radiation pressure are in general capable of providing the necessary inputs to prevent the over-production of extremely massive galaxies.

Aside from limiting the overall mass of galaxies, feedback is commonly invoked to explain a wide variety of observed galaxy properties, ranging from the creation and maintenance of the red sequence, the stellar mass to halo mass relation, the correlation between supermassive black hole mass and stellar velocity dispersion, the mass-metallicity relation, the normalization of the Kennicutt-Schmidt star formation 'law', and the presence of vast quantities of metal-enriched gas in the circumgalactic medium surrounding galaxies (e.g. Fabian 2012; Hopkins et al. 2014; Kormendy \& Ho 2013; Werk et al. 2014; Kennicutt \& Evans 2012).

From a theoretical standpoint, feedback has been difficult to implement in hydrodynamical simulations as the spatial and temporal scales of the energy/momentum deposition are much smaller than the typical resolution limit of simulations. At the highmass end, high-resolution hydrodynamical simulations currently favor dramatic processes such as merger-induced starbursts and subsequent supermassive black hole accretion to 
provide enough energy to heat and expel the gas supply and stave off further star formation (e.g. Hopkins et al. 2008; Ceverino et al. 2015; Wellons et al. 2015). Feedback also plays an important role for less extreme galaxies. Recent simulation work with novel prescriptions for feedback yields galaxies with highly time-variable star formation rates (SFRs) as the star-forming gas is temporarily disrupted before being able to cool and resume forming stars (e.g. Hopkins et al. 2014).

Massive galactic winds or outflows are a nearly-ubiquitous signature of feedback in nearby and intermediate-redshift galaxies. One challenge is that outflows are inherently multiphase in structure, spanning five or more orders of magnitude in gas temperature and density even within a single galactic wind (e.g., Scannapieco 2013; Leroy et al. 2015; Schneider \& Robertson 2017). Powerful outflows have been observed in hot ionized plasma, 'warm' ( $\left.T \sim 10^{4} \mathrm{~K}\right)$ ionized gas (e.g. Förster Schreiber et al. 2014; Genzel et al. 2014), and cooler neutral atomic gas (e.g. Shapley et al. 2003; Rupke et al. 2005). Most intriguing, however, has been the discovery of ubiquitous massive molecular winds in low-redshift galaxies, as this may indicate that feedback is capable of suppressing star formation by acting directly on the gas from which stars form (e.g., Feruglio et al. 2010; Veilleux et al. 2013; Cicone et al. 2014).

Because the spectral signatures of outflows are very faint, few detections of cold atomic/molecular outflows exist at high redshifts (e.g., Cicone et al. 2015; Spilker et al. 2018). Nevertheless, the $z>1$ era represents an important epoch in the history of the universe, probing the early and rapid formation of the first massive galaxies, the creation of the first passive 'red-and-dead' objects, and the peak epoch of cosmic star formation (e.g. Straatman et al. 2014; Madau \& Dickinson 2014). The completion of ALMA now allows for cold galactic winds to be detected and in some cases spatially resolved into the distant universe, while JWST will enable detections of the warm ionized phase of outflows beyond $z=2$.

\section{Plausible Long-Wavelength Outflow Tracers for the High-Redshift Universe}

Based on lessons learned from extensive observational investment in the nearby universe, a number of plausible outflow tracers are accessible by ALMA in the distant universe. In most cases it is the vastly increased sensitivity of ALMA (rather than spatial resolution or receiver frequency coverage) that makes these tracers observable. I also note that most of these tracers are also accessible to other sub-mm/mm interferometers such as the SMA and NOEMA, although the lower sensitivity of these facilities makes detections more difficult. This is not an exhaustive list of possible outflow tracers, instead only including those most commonly observed.

Hydroxyl (OH). Far-infrared lines of $\mathrm{OH}$ are perhaps the best and most reliable tracer of molecular outflows available. Compared to the other available tracers of molecular outflows, $\mathrm{OH}$ offers several advantages (as well as some disadvantages). First, the presence of winds is manifest through blueshifted absorption features against the bright dust continuum of the host galaxy, an unambiguous signature of outflowing material. This is particularly relevant in the high-redshift universe, where the violent processes of galaxy accretion and assembly can lead to emission at high velocities relative to the systemic that is unrelated to galactic outflows (e.g. accreting subhalos or major and minor mergers; Narayanan et al. 2015). Second, its ground-state transitions that trace the bulk of the molecular material lie at rest-frame wavelengths near the peak of the dust spectral energy distribution, $\sim 100 \mu \mathrm{m}$. This allows for observations of a given duration to reach better levels of contrast with the continuum compared to longer-wavelength molecular lines, the relevant quantity for absorption line work. Finally, due to its high dipole moment, the absorption line transitions of $\mathrm{OH}$ are strong and sensitive even to relatively small 
amounts of molecular material. For example, the timescale for spontaneous emission of the ground-state $119 \mu \mathrm{m}$ transitions of $\mathrm{OH}$ is $\sim 100 \mathrm{~s}$, compared to $\sim 10^{7} \mathrm{~s}$ for carbon monoxide. This ensures that the vast majority of the $\mathrm{OH}$ molecules are in the ground state and available to absorb continuum photons.

The strength of its transitions is also one of the primary drawbacks of $\mathrm{OH}$ - the lines become optically thick even for low column densities of gas, requiring additional lower-opacity lines to be observed to improve estimates of, e.g., the mass outflow rate. Another drawback of $\mathrm{OH}$ is that the transitions most useful for observing outflows are at short rest-frame wavelengths $(<120 \mu \mathrm{m})$, and so can only be observed by ground-based interferometers at redshifts $z>2$ (and practically only $z>4$ given weather requirements for high-frequency work). Future far-IR facilities such as the Origins Space Telescope will be able to detect these lines in virtually every star-forming galaxy out to $z \sim 3$.

The Herschel/PACS instrument observed multiple transitions of $\mathrm{OH}$ in nearby quasar host galaxies and Ultra/Luminous Infrared Galaxies (U/LIRGs), finding evidence for ubiquitous outflows (e.g. Veilleux et al. 2013; González-Alfonso et al. 2017). The high detection rate, $\sim 70 \%$ of sources with detected outflows, implies that both the intrinsic occurrence rate and the covering fraction / opening angle of these outflows must be very high (or they could not be detected in absorption). Currently the most distant unambiguous outflow is presented by Spilker et al. (2018) in a highly dust-obscured star-forming galaxy at $z=5.3$, discussed in more detail below.

Carbon Monoxide ( $\mathrm{CO}$ ). Lines of $\mathrm{CO}$ have long been used as tracers of molecular gas in galaxies because it is both a very abundant molecule and its transitions are easily accessible in the local universe with ground-based millimeter telescopes (e.g. Wilson et al. 1970). For CO the outflow signature manifests as excess emission at high relative velocities to the host systemic velocity. In the nearby universe where it is possible to resolve the $\mathrm{CO}$ emission from galaxies at high resolution, it is also possible to distinguish the emission associated with outflows kinematically, as the outflowing material is not expected to follow the predominant galactic kinematics (e.g. launched from the minor axis, perpendicular to the overall rotation curve). The CO emission associated with outflows is typically $>10 \times$ fainter than the galaxy itself, requiring very deep observations in order to detect and characterize outflows this way. A compilation of local-universe CO outflow detections can be found in Fluetsch et al. (2019). Reassuringly, the results from $\mathrm{CO}$ observations of outflows generally agree with those from $\mathrm{OH}$ despite very different assumptions and modeling approaches (Lutz et al. in prep.).

At high redshift $\mathrm{CO}$ remains a difficult tracer of outflows. The ground-state $\mathrm{CO}(1-0)$ transition is generally not accessible, requiring observations to target a more highlyexcited transition that may not trace the bulk of the molecular material. Like $\mathrm{OH}$, transitions of $\mathrm{CO}$ are also optically thick, requiring an estimate of a conversion factor to translate the observed luminosity to an outflowing mass (the infamous $\mathrm{CO}-\mathrm{H}_{2}$ conversion factor, see Bolatto et al. 2013 for a review). Nevertheless, some recent studies suggest detections of high-velocity $\mathrm{CO}$ emission possibly signaling molecular outflows (e.g. Brusa et al. 2018; Herrera-Camus et al. 2019). Future facilities such as the next generation Very Large Array (ngVLA) should be able to detect low-excitation transitions of CO in outflows for 'normal' star-forming galaxies out to $z>3$ (Spilker \& Nyland 2018).

Ionized Carbon $\left(C^{+}\right)$. As typically the brightest far-IR or millimeter line in galaxies, the $158 \mu \mathrm{m}$ transition of [CII] has become a workhorse for high-redshift observations, detectable by ALMA even in 'normal' galaxies with star formation rates $<100 \mathrm{M}_{\odot} / \mathrm{yr}$ in a few hours or less. [CII] emission arises in gas with a wide variety of temperatures and densities, including [H $\left.\mathrm{H}_{\mathrm{II}}\right]$ regions, the largely-neutral ISM, and the outer parts of molecular clouds. This also means that it is difficult to disentangle the contributions to the total luminosity from any of these individual sources; outflows detected in [CiI] likely 
trace some mixture of both cool atomic and molecular gas in the winds. As with CO, the signature of outflows from [CII] is excess emission in high-velocity wings of the line profile that is much fainter than the host galaxy. Janssen et al. (2016) present an analysis of Herschel observations, comparing outflow properties from [CiI] with those from $\mathrm{OH}$ absorption in the same galaxies, finding that nearly all galaxies would be characterized as showing outflow in both tracers and with a consistent outflow mass from both tracers.

At high redshift the use of [CII] as an outflow tracer remains controversial. Cicone et al. (2015) present PdBI observations of a $z=6.4$ quasar that shows tentative evidence of [CII] wings extending up to $1400 \mathrm{~km} \mathrm{~s}^{-1}$ relative to the galaxy, although this line emission therefore fills nearly the entire PdBI bandpass making continuum level estimation more difficult. The advent of far more sensitive ALMA observations has not settled this debate, with different groups finding evidence either for or against [CII] outflows from stacked spectra of the same sample of galaxies (Decarli et al. 2018; Gallerani et al. 2018), where the former strongly rules out an outflow with the same brightness as that claimed by Cicone et al. (2015); none of the sources individually show evidence for outflows. It is also interesting to note that we do not detect an outflow in [CII] in any of the objects discussed further below despite very high signal-to-noise and clear and obvious outflows seen in $\mathrm{OH}$. At present, then, the evidence that [CII] can or should be used as an outflow tracer is mixed at best $-[\mathrm{CII}]$ does not appear to be a reliable outflow tracer.

\section{Spatially-Resolved Molecular Winds from the South Pole Telescope Survey}

The South Pole Telescope collaboration has been conducting a survey of molecular outflows traced by $\mathrm{OH}$ in a sample of $z>4$ gravitationally lensed dusty, star-forming galaxies. Due to its long selection wavelength and flux cutoff that mostly selects lensed sources, the redshifts of this sample are typically much higher than 'traditional' single-dish $870 \mu \mathrm{m}$ selected sources, with $z_{\text {median }} \sim 4$ extending to $z \approx 7$ (Weiß et al. 2013; Béthermin et al. 2015; Strandet et al. 2016; Marrone et al. 2018). The brightness of these sources and the availability of pre-existing gravitational lens models (Spilker et al. 2016) makes this sample ideal for detections of molecular outflows via $\mathrm{OH}$ absorption.

The first object from this sample was recently published (Figure 1, Spilker et al. 2018), and this detection currently stands as both the highest-redshift and only spatiallyresolved molecular outflow in the distant universe. In that work, we found an outflow that reaches speeds up to $800 \mathrm{~km} \mathrm{~s}^{-1}$ relative to the galaxy's systemic redshift (determined from [CII]). A lensing reconstruction showed that the outflow covers a large fraction of the source $\approx 80 \%$, in rough agreement with the overall (spatially unresolved) detection fraction of low-redshift U/LIRGs from Herschel. The lensing analysis also showed that while the continuum emission, corresponding to obscured star formation in the galaxy, was fairly smooth, the outflowing material showed signs of clumpiness on kiloparsec scales. From simple assumptions about the $\mathrm{OH}$ optical depth and outflow geometry, we determined that the outflow rate of molecular material alone was within a factor of 2 of the star formation rate, even without including any contributions from warmer neutral or ionized gas. This implies that outflows can be efficient at removing the star-forming gas from high-redshift galaxies, and possibly links these highly star-forming galaxies to early massive quiescent galaxies observed by $z \sim 4$ (Straatman et al. 2014).

We have subsequently been working to extend this work to a large sample of $z>4$ galaxies with spatially-resolved outflows (Spilker et al. in prep.). Even before any lensing analysis it is already clear that outflows must be nearly ubiquitous among dusty starforming galaxies at high redshift - we have detected an unambiguous outflow in nearly 

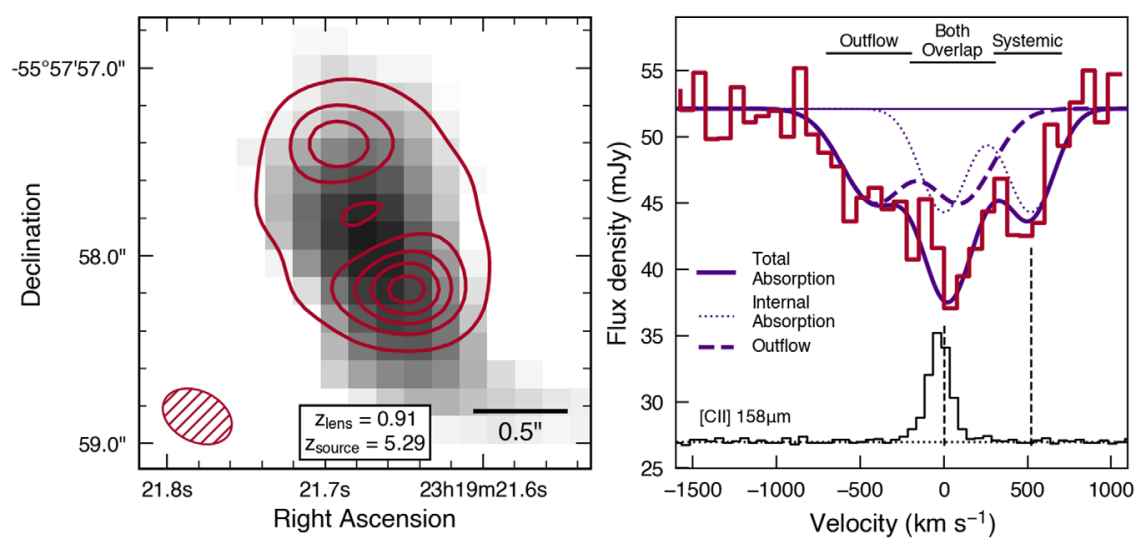

Figure 1. ALMA observations of the molecular outflow in the $z=5.3$ galaxy SPT2319-55. Left: ALMA rest-frame $119 \mu \mathrm{m}$ dust continuum observations (red contours) on a K-band image of the foreground lensing galaxy (grayscale). Right: The apparent spectrum (not corrected for lensing magnification) of the $\mathrm{OH} 119 \mu \mathrm{m}$ doublet transitions, with vertical dashed lines indicating the velocities of the two doublet components. A [CII] spectrum is also shown with arbitrary scaling as an indication of the line width of this galaxy; we seen no evidence for [CII] emission associated with the outflow. The spectrum is fit with two components representing systemic absorption from gas within the galaxy as well as a strongly blueshifted component representing the molecular outflow, which reaches velocities up to $\approx 800 \mathrm{~km} \mathrm{~s}^{-1}$. Figure adapted and reproduced with permission from Spilker et al. (2018).

every source thus far observed. As with the low-redshift Herschel results, this again must imply that the occurrence rate of molecular winds must be nearly unity. The fact that the outflows are detected in absorption also implies that either the opening angle of these outflows must also be near unity, or that the outflows typically exhibit some preferred geometry such that they are capable of absorbing photons along nearly all lines of sight (e.g., spherically expanding from the nuclear regions). Lensing reconstructions should be able to determine which of these possibilities is correct, if not both.

\section{Future Prospects: ALMA and JWST}

The outflows described in the previous section were nearly trivial to detect and spatially resolve with ALMA - no source was observed for longer than 30min. Thus it should be possible to push the study of outflows from high-redshift sources to either intrinsically less-luminous (but still lensed) sources, and/or to unlensed (but intrinsically very luminous) sources with observation times of a few hours per source or less.

Our observations only reached spatial resolutions of $\approx 0.3-0.5^{\prime \prime}$, nowhere near the maximum possible spatial resolution achievable by ALMA. Future directions could thus include detecting outflows at low spatial resolution and then resolving them on scales of a few hundred parsecs in order to determine the detailed outflow geometry. This is of particular interest for two main reasons. First, while it is possible to directly follow the mass in outflows in simulations, it is very difficult to measure accurate outflow rates from observations - every available tracer requires numerous assumptions in order to translate observed quantities into outflow masses or rates. The structure of outflows, on the other hand, can be determined in both observations and simulations and directly compared. Second, simulations typically find it extremely challenging to produce fast molecular outflows as observed, because the molecular gas is rapidly shredded by hydrodynamical 
instabilities long before it can be accelerated to the observed velocities (e.g. Schneider et al. 2018). This has led some to suggest that the molecular material in outflows has possibly cooled and condensed from a hotter wind at large radii, after it has already been accelerated (e.g. McCourt et al. 2018; Richings \& Faucher-Giguère 2018). These two scenarios lead to very different predictions for the detailed structure of molecular outflows: in the former case the molecular gas is concentrated in the few small clumps that were dense enough to avoid destruction, while the latter case predicts a far more uniform 'fog' of parsec-scale molecular droplets that would be unresolvable by any observation.

The advent of the James Webb Space Telescope will also prove revolutionary for the study of galactic outflows at very high redshifts. JWST will allow for the first observations of the multi-phase nature of outflows at $z>4$ through deep integral field observations of $\mathrm{H} \alpha$ emission. $\mathrm{H} \alpha$ has been shown to be a good tracer of the warm ionized phase of outflows out to $z \sim 2$, but is redshifted beyond the accessible atmospheric windows for the very early universe. The high spatial resolution, sensitivity, and wavelength coverage of the NIRSPEC instrument's integral field mode will allow observations of both the ionized and molecular phases of outflows on matched spatial scales, which has thus far only been possible for very nearby galaxies.

\section{References}

Béthermin, M., De Breuck, C., Sargent, M., \& Daddi, E. 2015, A\&AA, 576, L9

Bolatto, A. D., Wolfire, M., \& Leroy, A. K. 2013, ARA\&A, 51, 207

Brusa, M., Cresci, G., Daddi, E., et al. 2018, A\& A, 612, A29

Ceverino, D., Dekel, A., Tweed, D., \& Primack, J. 2015, MNRAS, 447, 3291

Cicone, C., Maiolino, R., Sturm, E., et al. 2014, A\&A, 562, A21

Cicone, C., Maiolino, R., Gallerani, S., et al. 2015, A\& $A$, 574, A14

Decarli, R., Walter, F., Venemans, B. P., et al. 2018, ApJ, 854, 97

Fabian, A. C. 2012, ARA $\& A, 50,455$

Feruglio, C., Maiolino, R., Piconcelli, E., et al. 2010, A\&A, 518, L155

Fluetsch, A., Maiolino, R., Carniani, S., et al. 2019, MNRAS, 483, 4586

Förster Schreiber, N. M., Genzel, R., Newman, S. F., et al. 2014, ApJ, 787, 38

Gallerani, S., Pallottini, A., Feruglio, C., et al. 2018, MNRAS, 473, 1909

Genzel, R., Förster Schreiber, N. M., Rosario, D., et al. 2014, ApJ, 796, 7

González-Alfonso, E., Fischer, J., Spoon, H. W. W., et al. 2017, ApJ, 836, 11

Herrera-Camus, R., Tacconi, L., Genzel, R., et al. 2019, ApJ, 871, 37

Hopkins, P. F., Hernquist, L., Cox, T. J., \& Kereš, D. 2008, ApJS, 175, 356

Hopkins, P. F., Kereš, D., Oñorbe, J., et al. 2014, MNRAS, 445, 581

Janssen, A. W., Christopher, N., Sturm, E., et al. 2016, ApJ, 822, 43

Kennicutt, R. C., \& Evans, N. J. 2012, ARA\&AA, 50, 531

Kormendy, J., \& Ho, L. C. 2013, ARA\&A, 51, 511

Leroy, A. K., Walter, F., Martini, P., et al. 2015, ApJ, 814, 83

Madau, P., \& Dickinson, M. 2014, ARA $\dot{\xi} A, 52,415$

Marrone, D. P., Spilker, J. S., Hayward, C. C., et al. 2018, Nature, 553, 51

McCourt, M., Oh, S. P., O'Leary, R., \& Madigan, A.-M. 2018, MNRAS, 473, 5407

Narayanan, D., Turk, M., Feldmann, R., et al. 2015, Nature, 525, 496

Richings, A. J., \& Faucher-Giguère, C.-A. 2018, MNRAS, 474, 3673

Rupke, D. S., Veilleux, S., \& Sanders, D. B. 2005, ApJS, 160, 115

Scannapieco, E. 2013, ApJ, 763, L31

Schneider, E. E., \& Robertson, B. E. 2017, ApJ, 834, 144

Schneider, E. E., Robertson, B. E., \& Thompson, T. A. 2018, ApJ, 862, 56

Shapley, A. E., Steidel, C. C., Pettini, M., \& Adelberger, K. L. 2003, ApJ, 588, 65

Spilker, J., \& Nyland, K. 2018, arXiv e-prints, arXiv:1810.06605

Spilker, J. S., Marrone, D. P., Aravena, M., et al. 2016, ApJ, 826, 112

Spilker, J. S., Aravena, M., Béthermin, M., et al. 2018, Science, 361, 1016 
Straatman, C. M. S., Labbé, I., Spitler, L. R., et al. 2014, ApJ, 783, L14

Strandet, M. L., Weiß, A., Vieira, J. D., et al. 2016, ArXiv e-prints, arXiv:1603.05094

Veilleux, S., Meléndez, M., Sturm, E., et al. 2013, ApJ, 776, 27

Weiß, A., De Breuck, C., Marrone, D. P., et al. 2013, ApJ, 767, 88

Wellons, S., Torrey, P., Ma, C.-P., et al. 2015, MNRAS, 449, 361

Werk, J. K., Prochaska, J. X., Tumlinson, J., et al. 2014, ApJ, 792, 8

Wilson, R. W., Jefferts, K. B., \& Penzias, A. A. 1970, ApJ, 161, L43 